\title{
The ATLAS Muon Trigger Performance in pp collisions at $\sqrt{s}=8 \mathrm{TeV}$
}

\section{Tomoe Kishimoto* on behalf of the ATLAS collaboration}

Kobe University

E-mail: Tomee.Kishimotedecrn.ch

Muons in the final state are key signatures for physics analyses at the Large Hadron Collider (LHC) of various processes, such as discovery of the Higgs boson, searches for new phenomena, as well as the measurements of Standard Model processes. Efficient triggers on muons and precise measurements of its performance are essential for physics analyses using muons. High rejection power against huge backgrounds at LHC is extremely important at online trigger stage. The ATLAS experiment employs a multi-level trigger architecture that selects the events in three sequential steps of increasing complexity and accuracy to cope with this challenging task. This paper reports about performance of the ATLAS muon trigger by using proton-proton collision data at $\sqrt{s}=8 \mathrm{TeV}$ collected in the year 2012 .

The European Physical Society Conference on High Energy Physics -EPS-HEP2013

18-24 July 2013

Stockholm, Sweden

\footnotetext{
* Speaker.
} 


\section{Introduction}

Muons in the final state are distinctive signatures of many physics studies at LHC, such as discovery of the Higgs boson, searches for new phenomena, as well as the measurements of Standard Model processes. Efficient triggers on muons and precise measurements of its performance are essential for physics analyses using muons. In this paper the performance of the ATLAS muon trigger evaluated with proton-proton collision data collected in 2012 at $\sqrt{s}=8 \mathrm{TeV}$ is presented.

\section{Muon trigger algorithms}

The selection of events with muons by the trigger system is performed in three steps. The signals from fast-response muon trigger detectors are processed by custom built hardware to generate a Level 1 (L1) trigger. The L1 muon system has six programmable $p_{T}$ thresholds to label the muon candidate. In addition, the L1 trigger carries the detector position information where muon candidates were found. In the following steps, software based High Level Trigger (HLT), which is subdivided into the Level 2 (L2) trigger and the Event Filter (EF), reconstructs tracks in the vicinity of the detector region reported by the L1 trigger. The L2 trigger performs a fast reconstruction of muons with a simple algorithm. Then the EF makes use of the offline muon reconstruction software to refine the trigger decision using full detector information. In 2012, the $p_{T}$ thresholds of the primary muon trigger were $15 \mathrm{GeV}$ for $\mathrm{L} 1$ (L1_MU15) and $24 \mathrm{GeV}$ for EF (EF_mu24i_tight). Additionally, the isolation cut $\left(\frac{\sum p_{T}}{p_{T}^{\mu}}<0.12\right)$ was applied at the EF stage, where $p_{T}^{\mu}$ is the muon $p_{T}$ and $\sum p_{T}$ is sum of the all track's $p_{T}$ with $p_{T}>1 \mathrm{GeV}$ within $\sqrt{\Delta \eta^{2}+\Delta \phi^{2}}=0.2$ from the muon.

\section{Trigger performance}

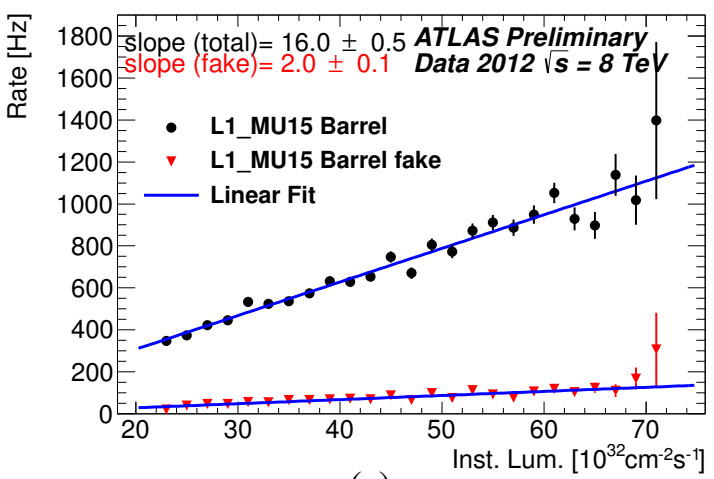

(a)

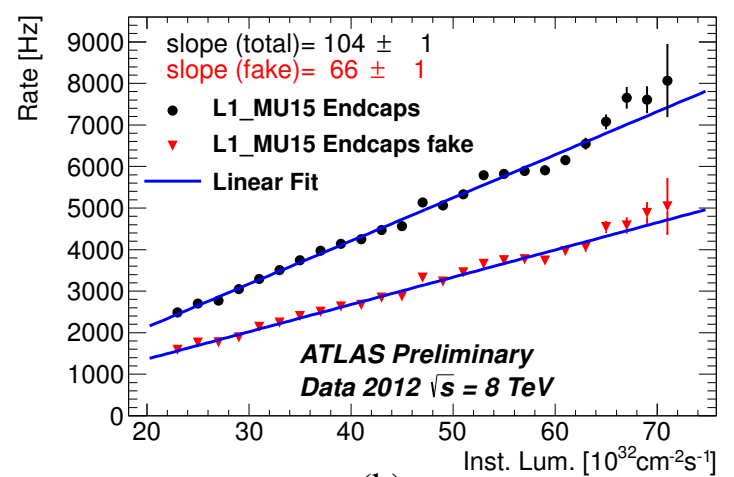

(b)

Figure 1: The L1 trigger rates in 2012 as a function of the instantaneous luminosity: (a) barrel and (b) endcaps regions. The black points represent the total measured rate, the red triangles the fake rates[1].

Figure 1 shows the $\mathrm{L} 1$ trigger rates in barrel $(|\eta|<1.05)$ and endcaps $(1.05<|\eta|<2.4)$ regions in terms of the instantaneous luminosity. The red triangles represent the fake rates defined as a trigger that does not match any offline reconstructed muon. The high fake fraction in the endcaps originates from particles peripheral to proton-proton collisions, for example protons in the beam 
halo and neutrons from nuclear interaction in the dense materials of e.g. magnets and beam shields. The fake triggers can be rejected by L2 trigger by requiring a matching track in the inner detectors. The L1 trigger rate scales linearly with the instantaneous luminosity, indicating that the L1 trigger rate is robust against pile-up.

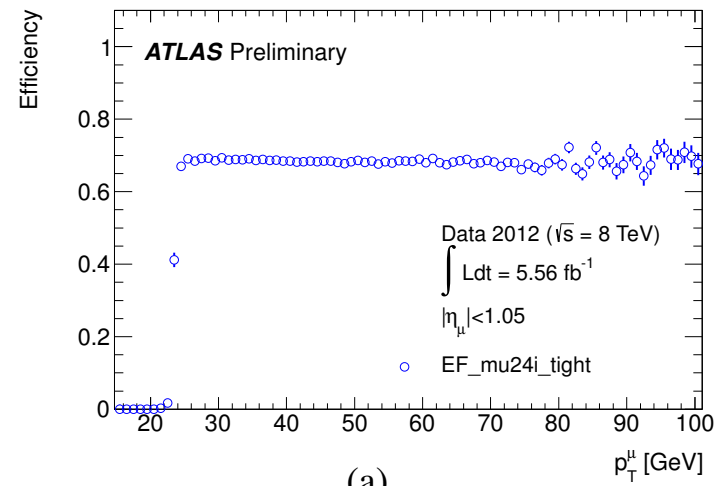

(a)

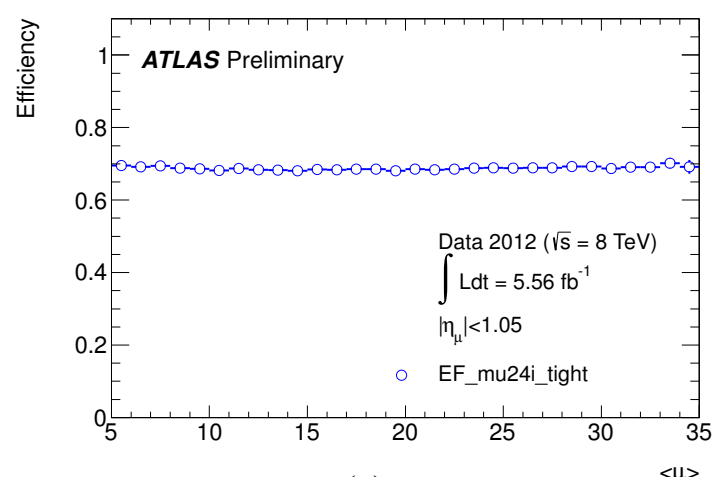

(c)

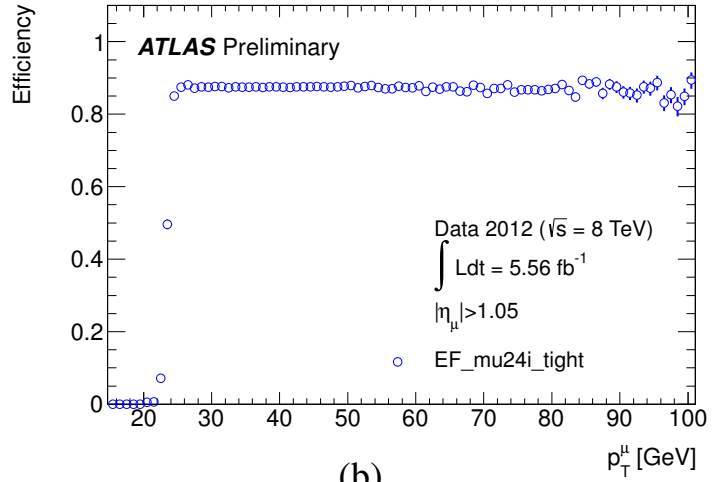

(b)

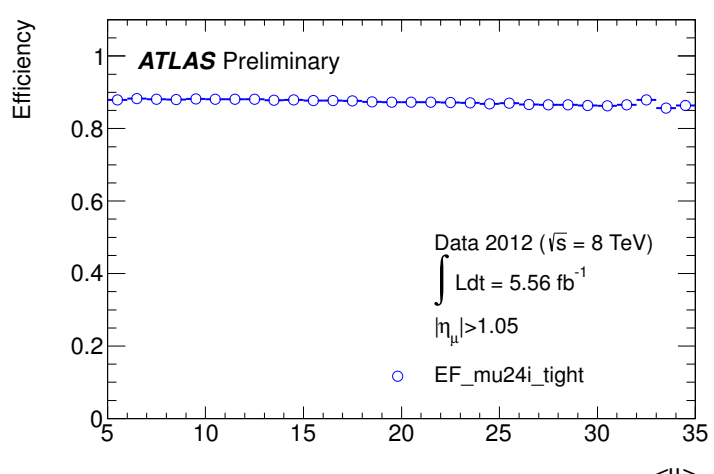

(d)

Figure 2: (a) and (b) show efficiencies of the EF trigger as a function of muon $p_{T}$ in the barrel and endcaps regions. (c) and (d) show the efficiencies as a function of number of interactions per bunch crossing[1].

The efficiencies of the muons trigger have been evaluated with the tag-and-probe method using samples containing pairs of muons from the decay of $Z$ bosons [2]. Figures 2(a) and 2(b) show the $p_{T}$ dependence of efficiencies. The efficiencies in the plateau region were about $70 \%$ in the barrel and $90 \%$ in the endcaps regions. The inefficiencies are due to limited geometrical acceptance and the hit efficiency of the L1 trigger detectors. Figures 2(c) and 2(d) show the trigger efficiencies in the plateau region as a function of interactions per bunch crossing. The efficiencies are stable in high- $p_{T}$ regions and show no significant pile-up dependence.

\section{References}

[1] https://twiki.cern.ch/twiki/bin/view/AtlasPublic/MuonTriggerPublicResults

[2] ATLAS Collaboration, Muon reconstruction efficiency in reprocessed 2010 LHC proton-proton collision data recorded with the ATLAS detector, ATLAS-CONF-2011-063.

[3] ATLAS Collaboration, The ATLAS Experiment at the CERN Large Hadron Collider, Journal of Instrumentation 3, S08003 (2008) 1-437. 